\title{
Pitfalls in respiratory monitoring of premature infants during kangaroo care
}

\author{
Dieter Sontheimer, Christine B Fischer, Frank Scheffer, Daisy Kaempf, \\ Otwin Linderkamp
}

\begin{abstract}
The reliability of respiratory monitoring, using either chest or back electrodes, was studied in 13 preterm infants during kangaroo care (infant-parent skin to skin contact). In three out of four infants with chest electrodes both infant and parental respiration were clearly visible on pneumograms. In these infants apnoeic pauses were not registered because parental respiration was recorded as infant breathing. Bradycardia and oxygen saturation were, however, properly registered. In infants with electrodes placed on the back infant respiration was less superimposed by parental breathing. However, even in some of these infants parental respiration was visible in the pneumograms.

It is concluded that during kangaroo care the electrodes should be placed on the back and monitoring should always include heart rate and oxygen saturation. (Arch Dis Child 1995; 72: F115-F117)
\end{abstract}

Keywords: premature infant, kangaroo care, respiratory monitoring.

Division of

Neonatology,

Department of

Pediatrics, University

of Heidelberg, Im

Neuenheimer Feld 150 ,

D-69120 Heidelberg,

Germany

D Sontheimer

C B Fischer

F Scheffer

D Kaempf

O Linderkamp

Correspondence to:

Dr Sontheimer.

Accepted 14 November 1994

Respiratory monitoring based on measurement of transthoracic impedance is the most widely used technique for recording respiratory patterns and detecting clinically important apnoea in premature infants. These monitors usually work by sending a high frequency electric current through electrodes placed on the infant's chest wall. The air:fluid ratio of the lung is altered by chest wall movements which change the transthoracic electric impedance. The reliability of respiratory monitoring of premature infants using transthoracic impedance has been evaluated in infants lying in an incubator or an open air crib. ${ }^{1}$

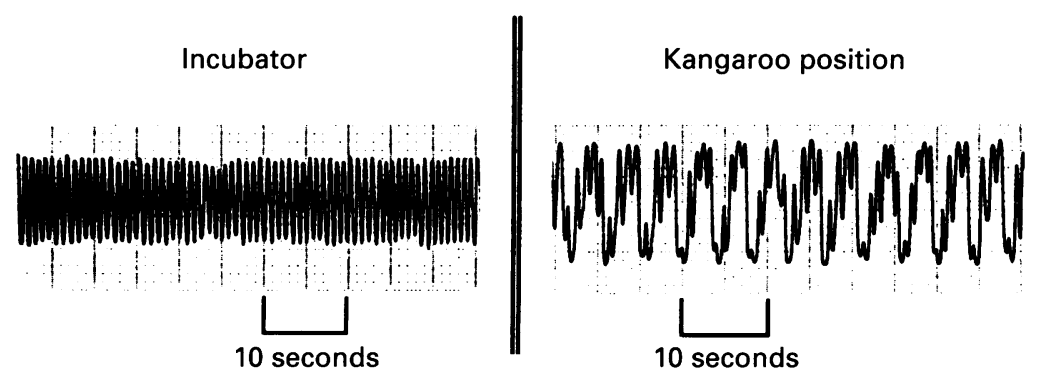

Figure 1 Respiratory patterns of regular breathing. Premature infant, gestational age 28 weeks, 30 days old, with electrodes placed on the chest. Superimposed paternal breathing patterns are evident during kangaroo care.
Kangaroo care (skin to skin contact between adult and infant) has recently been introduced in many neonatal intensive care units. ${ }^{23}$ During kangaroo care, naked premature infants wearing only a nappy are placed in an upright position on their mothers' or fathers' naked chests and covered with the parents' clothing and a blanket. Usually the child remains monitored. Different studies have indicated the feasibility of this method, and have found it to be free of risk and very enjoyable for mother and child. ${ }^{3-5}$ It may reduce apnoea and diminish periodic breathing. In our unit parents sit up to eight hours a day 'kangarooing' their infants.

In previous studies during kangaroo care we noticed that in some infants data on heart and breathing rate became unreliable because the respiratory pattern of the infant had been superimposed by maternal or paternal respiration. To our knowledge this phenomenon has not been described before and may produce misleading information during respiratory monitoring.

\section{Methods}

We recorded 26 pneumograms in 13 premature infants with a gestational age below 32 weeks, a birthweight under $1500 \mathrm{~g}$, and a postnatal age of less than 60 days. Infants were continuously monitored using an Edentec cardiorespiratory monitor $(2000 \mathrm{~W})$ based on transthoracic impedance and a Nellcor pulse oximeter (Eden Prairie). Heart rate, respiratory rate, and oxygen saturation were monitored continuously and periodic breathing, alarm and event data (apnoeas, bradycardias, tachycardias and oxygen desaturation) were also recorded. A chart recorder (Edentec 3700 multichannel charter) was also used for the continuous registration of heart rate, respiratory pattern, and oxygen saturation (figs 1-4).

In four infants the three electrodes for cardiorespirography were placed on the chest wall at the following sites: (1) second to third intercostal space, right midclavicular line; (2) second to third intercostal space, left midclavicular line; and (3) fifth to sixth intercostal space, left anterior axillary line. In the other nine infants the electrodes were placed at the left and right posterior axillary line and between the scapulae, to avoid direct contact between the electrodes and parental skin. Recordings were made two hours before, two hours during, and two hours after kangaroo care. 


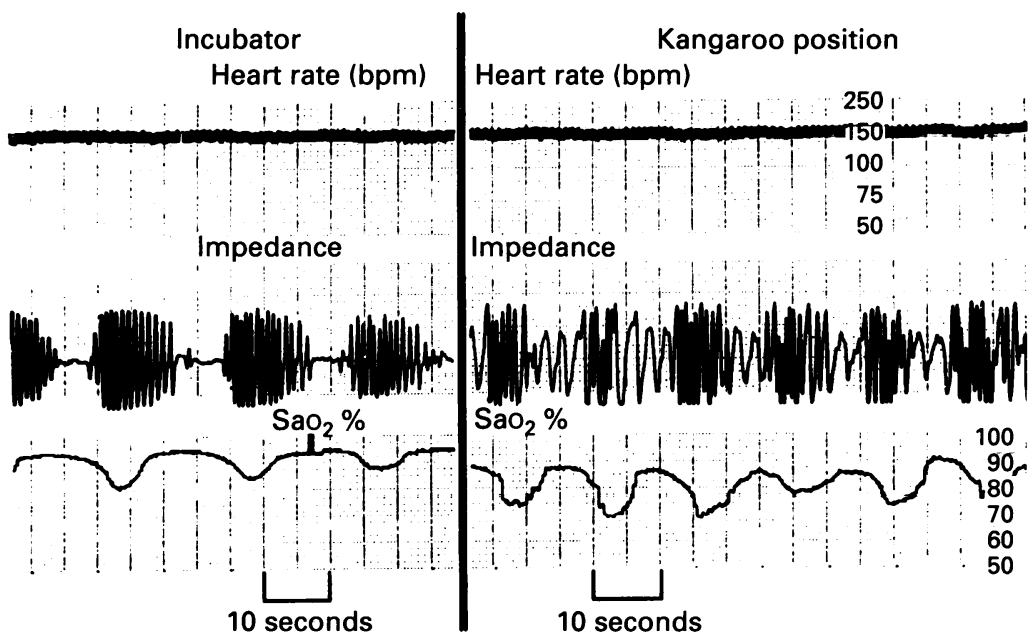

Figure 2 Cardiopneumogram showing pattern of periodic breathing. Premature infant, gestational age 26 weeks, 53 days old, with electrodes placed on the chest. Parental respiration visible in respiratory pauses of the infant during kangaroo care.

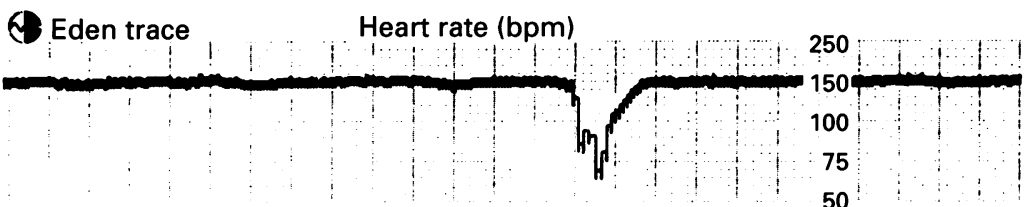

Impedance
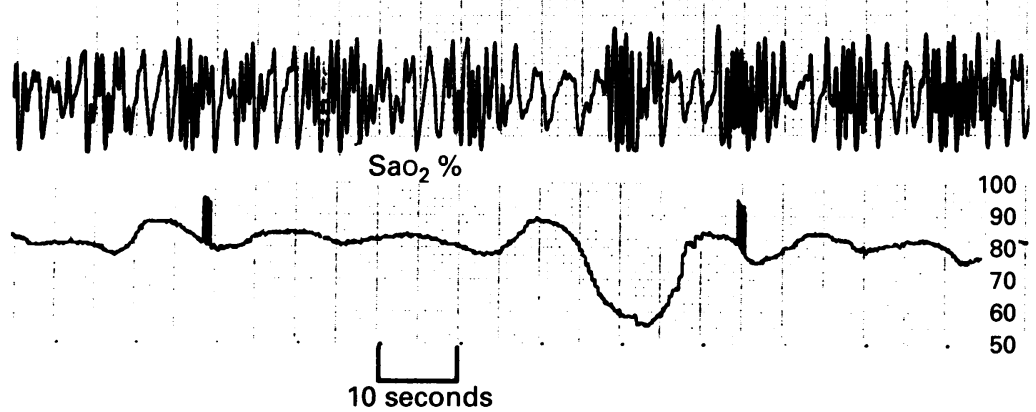

Figure 3 Non-detected apnoea during kangaroo care, with electrodes placed on the chest of the infant. Premaure infant, gestational age 26 weeks, 53 days old. Parental respiration recorded during infant apnoea followed by bradycardia and oxygen desaturation.

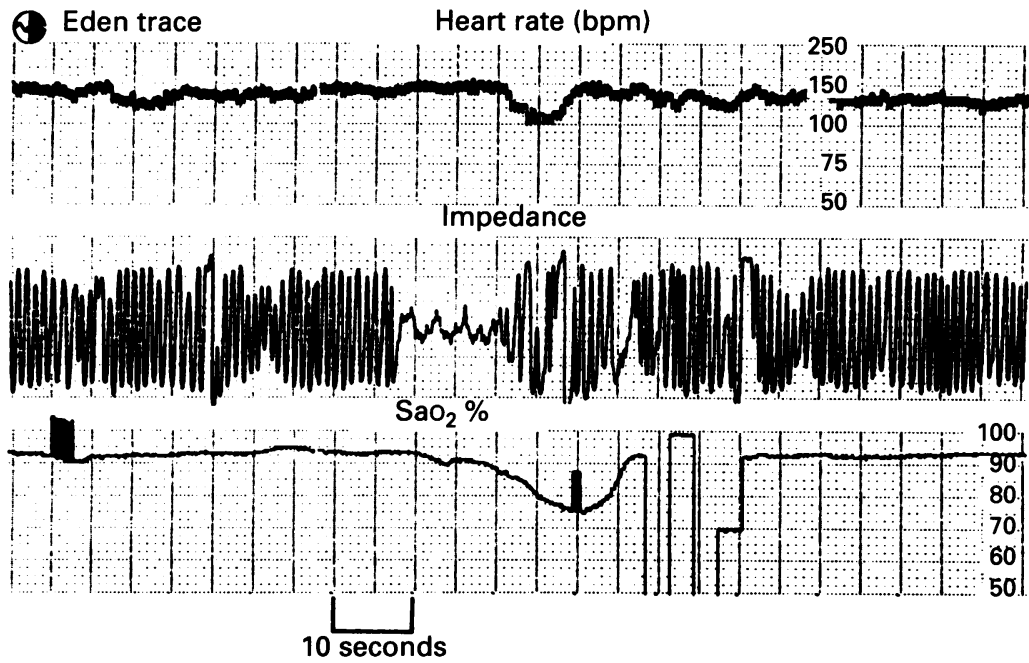

Figure 4 Parental respiration observed during apnoeas and periodic breathing. Apnoeas not recognised by the monitor.
Results

In three out of four infants with electrodes placed on the chest, both infant and parental breathing during kangaroo care were clearly visible on the pneumograms.

Fig 1 shows the respiratory patterns of an infant born at 28 weeks' gestational age, birthweight $1060 \mathrm{~g}$, at 30 days of age during kangaroo care with her mother. The respiratory pattern of the infant had been superimposed by maternal respiration. The infant's respiratory pattern is difficult to identify.

The cardiopneumogram shown in fig 2 shows a pattern of periodic breathing in a premature infant (gestational age 26 weeks, birthweight $640 \mathrm{~g}$ at 53 days old, study weight 1390 g) with chronic lung disease. While sleeping in the incubator, periodic breathing was observed and also documented by the event recorder. During skin to skin contact with the father, paternal respiration was clearly visible in the respiratory pauses of the infant. The monitor did not recognise the periodic breathing.

Fig 3 shows an apnoea during kangaroo care followed by bradycardia and oxygen desaturation. The apnoea lasted 13 seconds and the alarm threshold was set at 10 seconds, but the monitor did not recognise this event as paternal respiration was registered during the apnoea of the infant. Bradycardia and oxygen desaturation were, however, properly recorded.

In the other infant pairs with electrodes placed on the back respiratory patterns during kangaroo care were clearly identified as belonging to the infant and superimposed parental respiratory patterns were barely detected. However, parental respiration during apnoeas or periodic breathing were observed in the recordings of some infants (fig 4) and apnoeas were not always recognised as such by the monitor.

\section{Discussion}

We have shown that during kangaroo care parental respiratory patterns may be registered by transthoracic impedance measurement together with the infant's respiratory patterns. During skin to skin contact, the infant's thorax rises and falls as a result of parental breathing movements. Parental respiration can be registered by either passive chest movements of the infant or direct recording of parental impedance. When the infant is placed in a kangaroo position, the chest electrodes of the infant are in close contact with the moist paternal or maternal skin. In this way both the infant's and parent's impedance can be registered. Tactile stimulation by the parents during kangaroo care may also produce movement artefacts.

Periodic breathing and apnoeas may not be detected by transthoracic impedance monitors in some of the infants during kangaroo care. However, bradycardia and oxygen desaturation are reliably monitored (figs $2-4$ ). The reliability of the recording of pneumograms is improved by placing the electrodes on the back of the infant to avoid direct contact between the infant's electrodes and parental skin, but occasionally apnoeas are still not detected. 
Although our study shows that respiratory monitoring may not be reliable in some infants during kangaroo care, we still encourage parents to keep their infants in the kangaroo position as long as possible as we believe that the advantages of kangaroo care far outweigh the disadvantages. Previous studies have shown that respiratory monitoring can also fail to detect some types of apnoea (obstructive) ${ }^{6}$ and should, therefore, always be combined with monitoring heart rate and oxygen saturation.

We conclude that during kangaroo care electrodes should be placed on the back of the infant and that respiratory monitoring should be combined with monitoring heart rate and oxygen saturation.

1 Hunt CE. Cardiorespiratory monitoring. Clin Perinatol 1991;

2 Whitelaw A, Heisterkamp G, Sleath K, Acolet D, Richards M. Skin to skin contact for very low birthweight infants and
Mitelaw A, Heisterkamp $\mathrm{G}$, Sleath $\mathrm{K}$, Acolet $\mathrm{D}$, Richards their mothers. Arch Dis Child 1988; 63: 1377-81.

3 Anderson GC. Current knowledge about skin-to-skin (kangaroo) care for preterm infants. $\mathcal{f}$ Perinatol 1991; 11: 216-26.

4 De Leeuw R, Colin EM, Dunnebier EA, Mirmiran M. Physiological effects of kangaroo care in very small preterm infants. Biol Neonate 1991; 59: 149-55.

5 Affonso D, Bosque E, Wahlberg V, Brady JP. Reconciliation and healing for mothers through skin-to-skin contact provided in an American tertiary level intensive care nursery. Neonatal Network 1993; 12: 25-32.

6 Warburton D, Stark AR, Taeusch HW. Apnea monitor failure in infants with upper airway obstruction. Pediatrics 1977; 60: 742-4. 